\title{
Correspondence
}

\section{A CROSS CANADA SURVEY OF NEUROLOGISTS ON CONTROVERSIAL ISSUES IN EPILEPSY MANAGE- MENT}

To the Editor:

We have read with interest the special report "Epilepsy and Driving" by Andermann et al' and have found it to be an excellent general review of a complicated issue. It reviews the literature for the world as well as laws for different countries and finally those for each of the Canadian provinces. In the broad context many different scenarios arise for which one gets vague guidance from individual provincial guidelines ${ }^{2}$ on determining the fitness for driving. In an attempt to get a clear concensus on how neurologists approach certain potentially controversial driving issues Dr. Bruni and $\mathrm{I}^{3}$ did a survey. It was a broad survey on many issues but within it were questions that dealt specifically with driving related issues. In 1984 all neurologists in Canada were surveyed. The response rate was only $32 \%$ and thus we felt we could only look upon the results as showing an interesting trend as opposed to bringing a clear concensus to some of the thorny issues. Some relevant responses were: Only $8 \%$ automatically report all their patients with epilepsy to the provincial Motor Vehicles Branch. Reasons for not doing so included "not a watchdog", I am not a policeman", "not required", "it is the patient's responsibility", "I only report the real dangers", "most can be trusted", "only those in my opinion", etc. As Andermann et al summarize, in the majority of provinces it is obligatory to report patients with epilepsy.

Fifty-three percent of neurologists felt it could be safe for patients with simple partial seizures with full motor control and $50 \%$ of the neurologists felt that those with "auras" could probably also be allowed to drive. Provincial guidelines are remarkably vague on the definition of seizures and don't give clear direction to physicians on how to handle this situation. The phrase "in your opinion" is there in most instances allowing the physician the latitude to exercise their clinical judgment. Qualifying phrases or prudent advice received from this survey included "These partial seizures would not affect vision, would not be followed by obtundation or cloudy states or loss of consciousness and one ideally would like a witnessed description of the simple partial seizure or aura".

Eighty-one percent of neurologists felt that patients with alcohol withdrawal seizures should not drive and $60 \%$ felt that these individuals should be reported to the Motor Vehicles Branch in contrast to only $8 \%$ of neurologists who would routinely report people with epilepsy. Currently in Saskatchewan and Quebec such individuals are restricted from driving and B.C. is about to enact similar guidelines.

What started out to be a rather simple project, intended to get a clear concensus on some of these more contentious issues turned out to be a far more complicated matter. It quickly became apparent that there is no such thing as "an average case". Clearly the neurology community wanted flexibility to make recommendations for each individual case rather than being "hand-tied" by arbitrary guidelines that may be punitive or needlessly restrictive.

\author{
M.W. Jones \\ University Hospital; Shaughmessy Site \\ University of British Columbia \\ Vancouver; B.C.

J. Brumi
Wellesley Hospital
University of Toronto
Toronto, Ontario

I. Andermann F et al. Epilepsy and Driving, Can J Neurol Sci 1988; 15: $371-377$.

2. Guide for Physicians in Determining Fitness to Drive Motor Vehicles. Canadian Medical Association; Revised 1981; Ottawa. Ontario.

3. Jones M.W, Bruni J. A Cross-Canada Survey of Neurologists and Neurosurgeons on Controversial Issues in Epilepsy Management. Can J Neurol Sci 1985; 12: 174.

\section{PERIODIC COMPLEXES IN CREUTZFELDT JAKOB DISEASE AND SLEEP}

To the Editor:

Bilaterally synchronous periodic complexes (BPC) are seen in various disorders including Creutzfeldt Jakob disease (CJD), subacute sclerosing parencephalitis (SSPE), hypoxic encephalopathy, Binswanger disease and lipidoses.' Mechanisms involved in production of BPC remain unknown. We report 3 patients with CJD in whom BPC disappeared during sleep but reappeared on arousal.

Patient 1: This 69 year old female presented with memory loss, ataxia and incontinence of 4 months duration. Examination showed dementia, bilateral choreoathetosis, ataxia, spasticity and occasional myoclonus. She died 8 months later and diagnosis of CJD was verified at autopsy.

EEG showed background slowing and diffuse arrythmic delta. Di or triphasic BPC were seen every 600 milliseconds. During spontaneous drowsiness and sleep, BPC disappeared or diminished with suppression of amplitude. Following $2 \mathrm{mg}$. of intravenous diazepam, BPC disappeared for approximately 180 seconds and the patient appeared asleep during this period. On arousal, BPC reappeared.

Patient 2: This 62 year old patient presented with a three month history of progressive dementia, ataxia and incontinence. Examination also showed myoclonic jerks, spasticity and rigidity. She subsequently developed cortical blindness and dysphagia. Evoked potentials and CT scan of brain were normal. She died approximately a year after the onset of symptoms. Autopsy was refused.

EEG on admission showed diffuse arrythmic delta activity and diphasic BPC which occurred every 200 milliseconds with amplitude varying between 100 and 150 microvolts. BPC disappeared during spontaneous and chloral hydrate induced sleep but reappeared on arousal. Intravenous diazepam induced sleep and suppression of BPC for several minutes. Intravenous naloxone - which in opiate free individuals produces drowsiness ${ }^{2}$ - produced similar suppression of BPC.

Patient 3: a 68 year old, female presented with dementia, gait apraxia and aphasia of two months duration. Examination also showed bilateral dystonic posturing and myoclonic jerks. Her neurological condition rapidly deteriorated and she died two months after initial admission. 